\title{
UK Renal Registry 16th Annual Report: Appendix H Coding: Ethnicity, EDTA Primary Renal Diagnoses, EDTA Causes of Death
}

\section{H1: Ethnicity coding}

Ethnicity data is recorded in the clinical information systems in the individual renal centres in the format of $9 \mathrm{~S} \ldots$ read codes. If extracted from local PAS systems in a different format, it is recoded to the $9 \mathrm{~S}$... format by the centre, before being sent to the UK Renal Registry (UKRR). For report analyses, ethnic categories are condensed into five groups (White, South Asian, Black, Chinese and Other). For some analyses Chinese are grouped into Other.

\begin{tabular}{|c|c|c|c|c|}
\hline Read code & Ethnic category & Assigned group & Old PAS & New PAS \\
\hline 9S1. & White & White & 0 & $\mathrm{~A} 1$ \\
\hline 9SA9. & Irish (NMO) & White & & B1 \\
\hline 9SAA. & Greek Cypriot (NMO) & White & & CG \\
\hline 9SAB. & Turkish Cypriot (NMO) & White & & CJ \\
\hline 9SAC. & Other European (NMO) & White & & $\mathrm{C} 1$ \\
\hline $9 \mathrm{~S} 6$. & Indian & S Asian & 4 & H1 \\
\hline 9S7. & Pakistani & S Asian & 5 & J1 \\
\hline $9 S 8$. & Bangladeshi & S Asian & 6 & K1 \\
\hline 9SA6. & East African Asian & S Asian & & \\
\hline 9SA7. & Indian Subcontinent & S Asian & & \\
\hline 9SA8. & Other Asian & S Asian & & L1 \\
\hline 9S2. & Black Caribbean & Black & 1 & M1 \\
\hline $9 \mathrm{~S} 3$. & Black African & Black & 2 & N1 \\
\hline $9 S 4$. & Black/Other/non-mixed origin & Black & 3 & P1 \\
\hline 9S41. & Black British & Black & & PD \\
\hline 9S42. & Black Caribbean & Black & & \\
\hline $9 S 43$. & Black North African & Black & & \\
\hline $9 S 44$. & Black other African country & Black & & \\
\hline $9 S 45$. & Black East African Asian & Black & & \\
\hline 9S46. & Black Indian subcontinent & Black & & \\
\hline $9 S 47$. & Black Other Asian & Black & & \\
\hline $9 S 48$. & Black Black Other & Black & & $\mathrm{PE}$ \\
\hline $9 \mathrm{~S} 5$. & Black other/mixed & Black & & \\
\hline 9S51. & Other Black - Black/White origin & Black & & GC \\
\hline $9 S 52$. & Other Black - Black/Asian origin & Black & & GA \\
\hline $9 S 9$. & Chinese & Chinese & 7 & $\mathrm{R} 1$ \\
\hline 9T1C. & Chinese & Chinese & & \\
\hline 9SA. & Other ethnic non-mixed (NMO) & Other & & \\
\hline 9SA1. & British ethnic minority specified (NMO) & Other & & \\
\hline 9SA2. & British ethnic minority unspecified (NMO) & Other & & \\
\hline 9SA3. & Caribbean Island (NMO) & Other & & \\
\hline
\end{tabular}

\section{KARGER}

Fax +4161306 1234

E-Mail karger@karger.com

www.karger.com
(C) 2014 S. Karger AG, Basel

$1160-2110 / 14 / 1254-0353 \$ 38.00 / 0$

Accessible online at: www.karger.com/nec
UK Renal Registry, Southmead Hospital, Southmead Road, Bristol, BS10 5NB, UK

Email: renalregistry@renalregistry.nhs.uk 


\begin{tabular}{|c|c|c|c|c|}
\hline Read code & Ethnic category & Assigned group & Old PAS & New PAS \\
\hline 9SA4. & North African Arab (NMO) & Other & & \\
\hline 9SA5. & Other African countries (NMO) & Other & & \\
\hline 9SAD. & Other ethnic NEC (NMO) & Other & & S1 \\
\hline 9SB. & Other ethnic/mixed origin & Other & 8 & \\
\hline 9SB1. & Other ethnic/Black/White origin & Other & & E1 \\
\hline 9SB2. & Other ethnic/Asian/White origin & Other & & F1 \\
\hline 9SB3. & Other ethnic/mixed White origin & Other & & \\
\hline 9SB4. & Other ethnic/Other mixed origin & Other & & G1 \\
\hline
\end{tabular}

NMO denotes non-mixed origin

\section{H2: EDTA primary renal diagnoses}

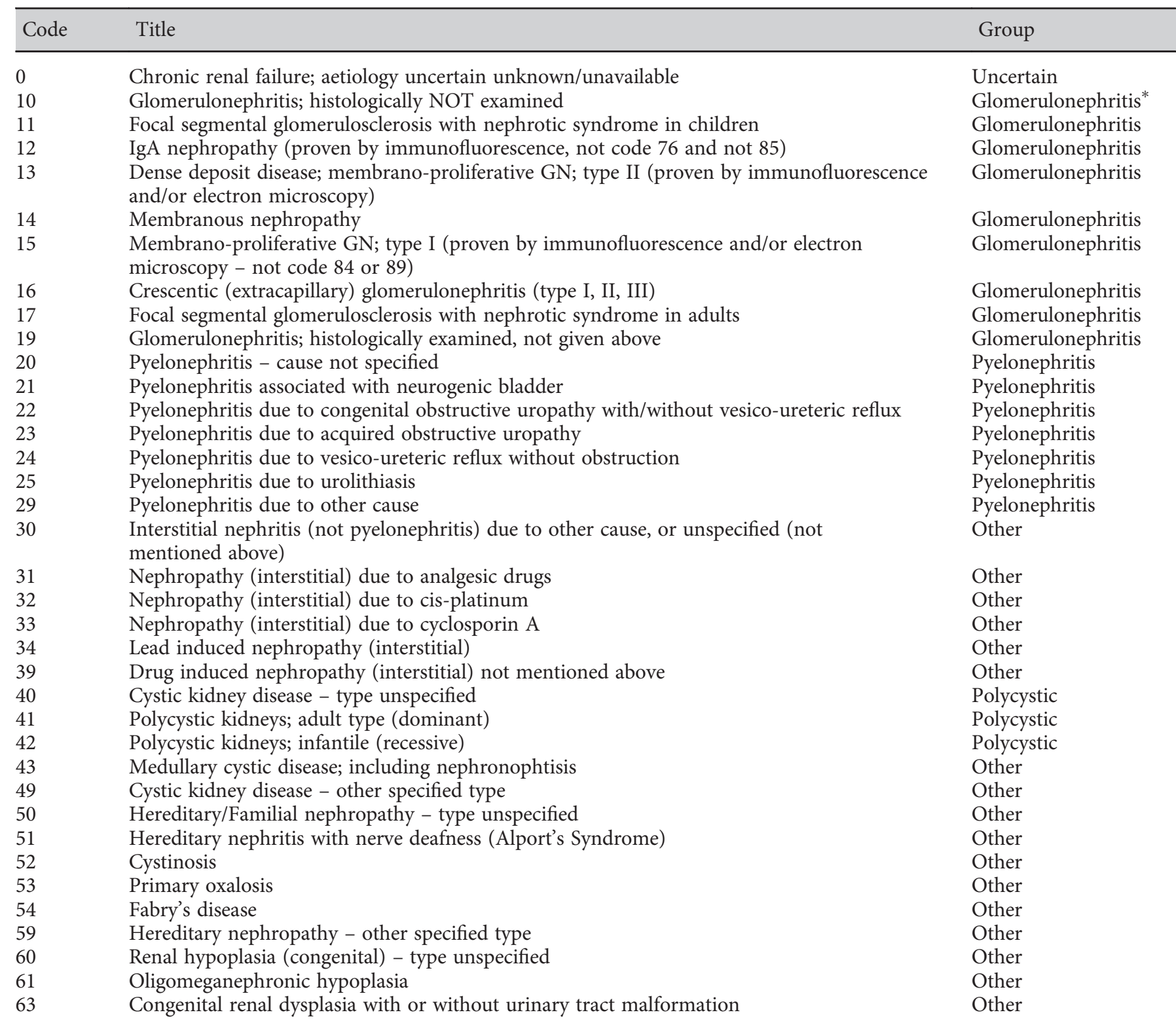




\begin{tabular}{lll}
\hline Code & Title & Group \\
\hline 66 & Syndrome of agenesis of abdominal muscles (Prune Belly) & Other \\
70 & Renal vascular disease - type unspecified & Renal vascular disease \\
71 & Renal vascular disease due to malignant hypertension & Hypertension \\
72 & Renal vascular disease due to hypertension & Hypertension \\
73 & Renal vascular disease due to polyarteritis & Renal vascular disease \\
74 & Wegener's granulomatosis & Other \\
75 & Ischaemic renal disease/cholesterol embolism & Renal vascular disease \\
76 & Glomerulonephritis related to liver cirrhosis & Other \\
78 & Cryoglobulinemic glomerulonephritis & Other \\
79 & Renal vascular disease - due to other cause (not given above and not code 84-88) & Renal vascular disease \\
80 & Type 1 diabetes with diabetic nephropathy & Diabetes \\
81 & Type 2 diabetes with diabetic nephropathy & Diabetes \\
82 & Myelomatosis/light chain deposit disease & Other \\
83 & Amyloid & Other \\
84 & Lupus erythematosus & Other \\
85 & Henoch-Schoenlein purpura & Other \\
86 & Goodpasture's syndrome & Other \\
87 & Systemic sclerosis (scleroderma) & Other \\
88 & Haemolytic Ureaemic Syndrome (including Moschcowitz syndrome) & Other \\
89 & Multi-system disease - other (not mentioned above) & Other \\
90 & Tubular necrosis (irreversible) or cortical necrosis (different from 88) & Other \\
91 & Tuberculosis & Other \\
92 & Gout nephropathy (urate) & Other \\
93 & Nephrocalcinosis and hypercalcaemic nephropathy & Other \\
94 & Balkan nephropathy & Other \\
95 & Kidney tumour & Other \\
96 & Traumatic or surgical loss of kidney & Other \\
98 & Not known & Missing \\
99 & Other identified renal disorders & Other \\
199 & Code not sent & Missing \\
\hline
\end{tabular}

*Prior to the 15th Annual Report categorised as 'uncertain' 


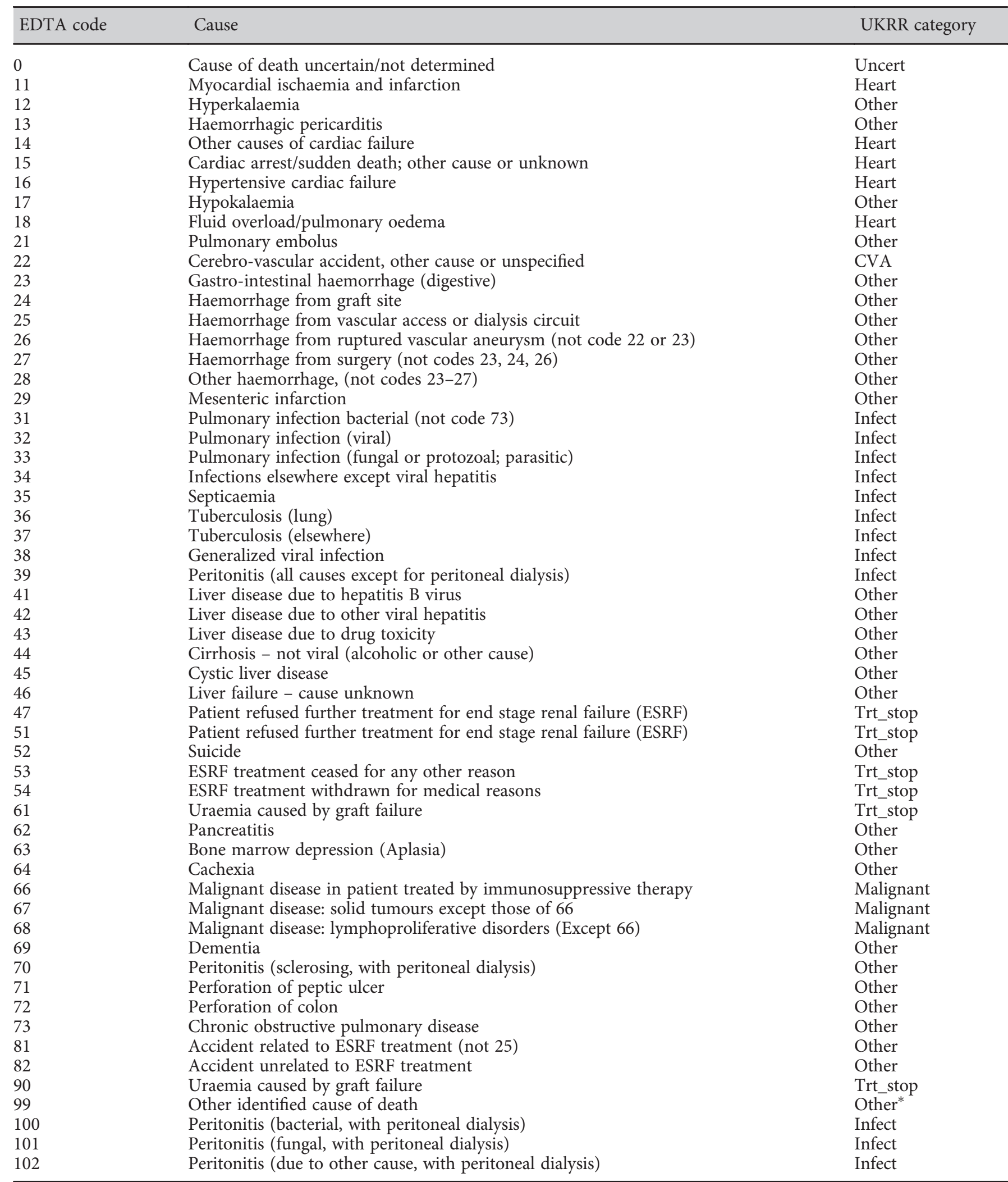

*Prior to the 15th Annual Report categorised as 'uncertain' 